\title{
Clinical and Angiographic Features in Three COVID-19 Patients with Takotsubo Cardiomyopathy. Case Report
}

\author{
Wolfgang Hoepler $^{1}$ (D) Marianna Theresia Traugott ${ }^{1}$ (D) $\cdot$ Guenter Christ ${ }^{2} \cdot$ Reinhard Kitzberger $^{1} \cdot$ Erich Pawelka $^{1}$ (D) \\ Mario Karolyi ${ }^{1}$ (D) Tamara Seitz ${ }^{1} \cdot$ Sebastian Baumgartner ${ }^{1} \cdot$ Hasan Kelani ${ }^{1} \cdot$ Christoph Wenisch $^{1} \cdot$ Hermann Laferl $^{1}$. \\ Alexander Zoufaly ${ }^{1}$ (D) $\cdot$ Lukas Weseslindtner $^{3} \cdot$ Stephanie Neuhold $^{1}$
}

Accepted: 28 November 2020 / Published online: 6 January 2021

(C) The Author(s), under exclusive licence to Springer Nature Switzerland AG part of Springer Nature 2021

\begin{abstract}
While coronavirus disease 2019 (COVID-19), caused by severe acute respiratory coronavirus 2 (SARS-CoV-2), has often been perceived as a predominantly respiratory condition, it is characterized by complications in multiple organ systems. Especially the involvement of the cardiovascular system, along with the possibly severe pulmonary injury, is crucial for prognosis. We identified three COVID-19 patients with takotsubo (TT) cardiomyopathy at our infectious diseases treatment center and present their clinical, laboratory, echocardiographic, electrocardiographic, and angiographic features. All patients were female (median age, 67 years); disease severity regarding COVID-19 ranged from asymptomatic to ARDS (adult respiratory syndrome) necessitating mechanical ventilation for 22 days. Angiography revealed normal coronary arteries in patient 1 , severe three-vessel coronary artery disease $(\mathrm{CAD})$ in patient 2 , and insignificant bystander $\mathrm{CAD}$ in patient 3 . All patients showed classic apical hypokinesia with basal hyperkinesia. In patient 3, TT cardiomyopathy resulted in transient cardiogenic shock. Twenty-eight-day mortality was $0 \%$ in this case series. In conclusion, takotsubo cardiomyopathy may be yet another clinical entity associated with SARS-CoV-2 infection.
\end{abstract}

Keywords COVID-19 · SARS-CoV-2 $\cdot$ Takotsubo cardiomyopathy $\cdot$ Stress cardiomyopathy $\cdot$ Coronary angiography

\section{Introduction}

Severe acute respiratory syndrome coronavirus 2 (SARS$\mathrm{CoV}-2$ ), the virus causing the ongoing coronavirus disease 2019 (COVID-19) pandemic, is continuing to baffle virologists and clinicians all over the world with a broad spectrum of manifestations in several different organ systems outside the respiratory tract.

Wolfgang Hoepler

wolfgang.hoepler@gmail.com;

wolfgang.hoepler@gesundheitsverbund.at

1 Fourth Medical Department with Infectious Diseases and Tropical Medicine, Klinik Favoriten/Kaiser-Franz-Josef Hospital, 1100 Vienna, Austria

2 Fifth Medical Department with Cardiology, Kaiser-Franz-Josef Hospital, 1100 Vienna, Austria

3 Center for Virology, Medical University of Vienna, 1090 Vienna, Austria
In a case-based review [1], the definition of COVID-19 as a multiorgan disease has been proposed, and viral affection of the cardiovascular system, the kidney, the liver, the nervous system, and, possibly, the reproductive system are described in this report.

Among the various complications of SARS-CoV-2 infection, cardiac injury is a particularly important and frequent clinical entity. In a Chinese cohort, up to $19.7 \%$ displayed cardiac involvement, which was an independent risk factor for in-hospital mortality [2].

In a study using more sophisticated methods such as cardiac magnetic resonance imaging, cardiac affection was diagnosed in a stunning $78 \%$ of patients who had recently recovered from COVID-19 [3].

Worryingly, myocarditis associated with SARS-CoV-2 has even been documented in young and healthy adults who had reported mild or no COVID-19 symptoms, potentially putting them at risk of arrhythmia and sudden cardiac death [4].

Arcari et al. [5] found a significant association between elevated cardiac biomarkers (troponin and natriuretic peptides) and indices of COVID-19 pneumonia severity. 
The pathomechanism for heart involvement in a disease due to a virus which had been believed to be a classic "respiratory" pathogen is incompletely understood. Several hypotheses have been proposed, such as the profound hypoxemia inducing myocardial ischemia [6], the cytokine storm associated with severe COVID-19 [7], an endothelialitis caused by SARS-CoV-2 not sparing the coronary arteries [8], and direct virus infection of pericytes as the cardiac target cells expressing a high density of ACE 2, the critical host cell receptor for SARS-CoV-2 [9].

Stress cardiomyopathy, or takotsubo cardiomyopathy (TT cardiomyopathy), may be an underreported complication of COVID-19.

TT cardiomyopathy is defined as an acute and reversible left ventricular systolic (and associated diastolic) dysfunction usually related to an emotionally or physically stressful event [10]. Moreover, the presence of left ventricular wall motion abnormalities is characteristically not limited to the supply area of a single coronary artery.

This report presents the clinical, echocardiographic, electrocardiographic, laboratory, and angiographic features of three COVID-19 patients with an additional diagnosis of stress cardiomyopathy.

Since the admission of the first patient with confirmed SARS-CoV-2 infection and adult respiratory distress syndrome to the intensive care unit at the end of February 2020 [11], our department of infectious diseases and tropical medicine has been serving as the primary treatment center for COVID-19 patients in Vienna, Austria.

Three COVID-19 patients with a diagnosis of takotsubo cardiomyopathy were identified between August and September 2020. Experienced cardiologists performed coronary angiography (CAG) to rule out coronary artery disease (CAD) or to diagnose bystander CAD in addition to TT cardiomyopathy.

We retrospectively analyzed the patients' clinical records.

Patient characteristics regarding clinical, echocardiographic, electrocardiographic, and angiographic hallmarks are presented in a descriptive manner; no statistical analysis was undertaken.

\section{Case Presentations}

Patient characteristics are summarized in Table 1.

All patients were female and had PCR-confirmed diagnosis of COVID-19.

Patient 1 was a 67-year-old woman who had presented to the emergency department with chest pain. An acute coronary syndrome was suspected and the patient was put on dual antiplatelet therapy (DAPT). However, coronary angiography (CAG) showed normal coronary arteries and a normally sized left ventricle with preserved ejection fraction (65\%) with severe hypo- to akinesia in parts of the apical and the inferoapical wall with hypercontractility of the basal segments of the heart.

Therefore, the diagnosis of TT cardiomyopathy was established and DAPT was stopped.

Her initial electrocardiogram (ECG) had shown complete right bundle branch block (QRS $160 \mathrm{~ms}$ ) with T wave inversions in leads I, aVL, and V3-V6. Three days later, the QRS complex was normal again but $\mathrm{T}$ wave inversions were more pronounced.

A marked rise in troponin levels (maximum $314.6 \mathrm{ng} / \mathrm{L}$, reference $0-58.9$ ) with levels of creatine kinase was noted, but troponin consecutively fell to $26 \mathrm{ng} / \mathrm{L}$ before discharge.

When the routine nasopharyngeal swab yielded a positive PCR result for SARS-CoV-2, the patient was transferred to our department for isolation purposes and for further management.

Transthoracic echocardiography 2 days after CAG showed a normal left ventricular function with mild apical akinesia and mild to moderate mitral insufficiency.

As far as COVID-19 symptoms were concerned, she remained asymptomatic during her entire stay at hospital and her chest X-ray was unremarkable.

The patient developed no complications and was discharged home in a very good general condition 14 days after CAG.

Follow-up echocardiography as an outpatient about a month after discharge showed normal left ventricular function without any regional differences and normal heart valves.

Patient 2 was a 60 -year-old patient admitted to our department because of dyspnea due to confirmed COVID-19. She was severely compromised regarding respiration, as she suffered from high-grade COPD and was still an active smoker with a history of 150 pack years, but responded well to extensive bronchospasmolytic therapy and did not require respiratory support apart from oxygen insufflation.

As the ECG routinely performed at admission was remarkable for ST-elevations and negative $\mathrm{T}$ waves in leads $\mathrm{V} 4$ and $\mathrm{V} 5$ and isolated negative T waves in leads II, III, aVF, and V6 (the patient later developed persistent $\mathrm{T}$ wave inversions in II, III, aVF, and V2 to V6), she received dual antiplatelet therapy with aspirin and clopidogrel plus heparin and was transferred to our intensive care unit for monitoring. Additionally, the laboratory workup showed marked elevations of troponin (maximum $333 \mathrm{ng} / \mathrm{L}$ ), Creatine Kinase (maximum $532 \mathrm{U} / \mathrm{L}$ ), and NT-pro-BNP (maximum $7275 \mathrm{ng} / \mathrm{L})$.

Echocardiography demonstrated moderately reduced systolic function and apical, anterior, and posterolateral akinesia.

CAG finally revealed severe three-vessel coronary artery disease (CAD), but also TT cardiomyopathy with classic apical ballooning and hyperkinesia of the basal segments (Figs. 1 and 2). 


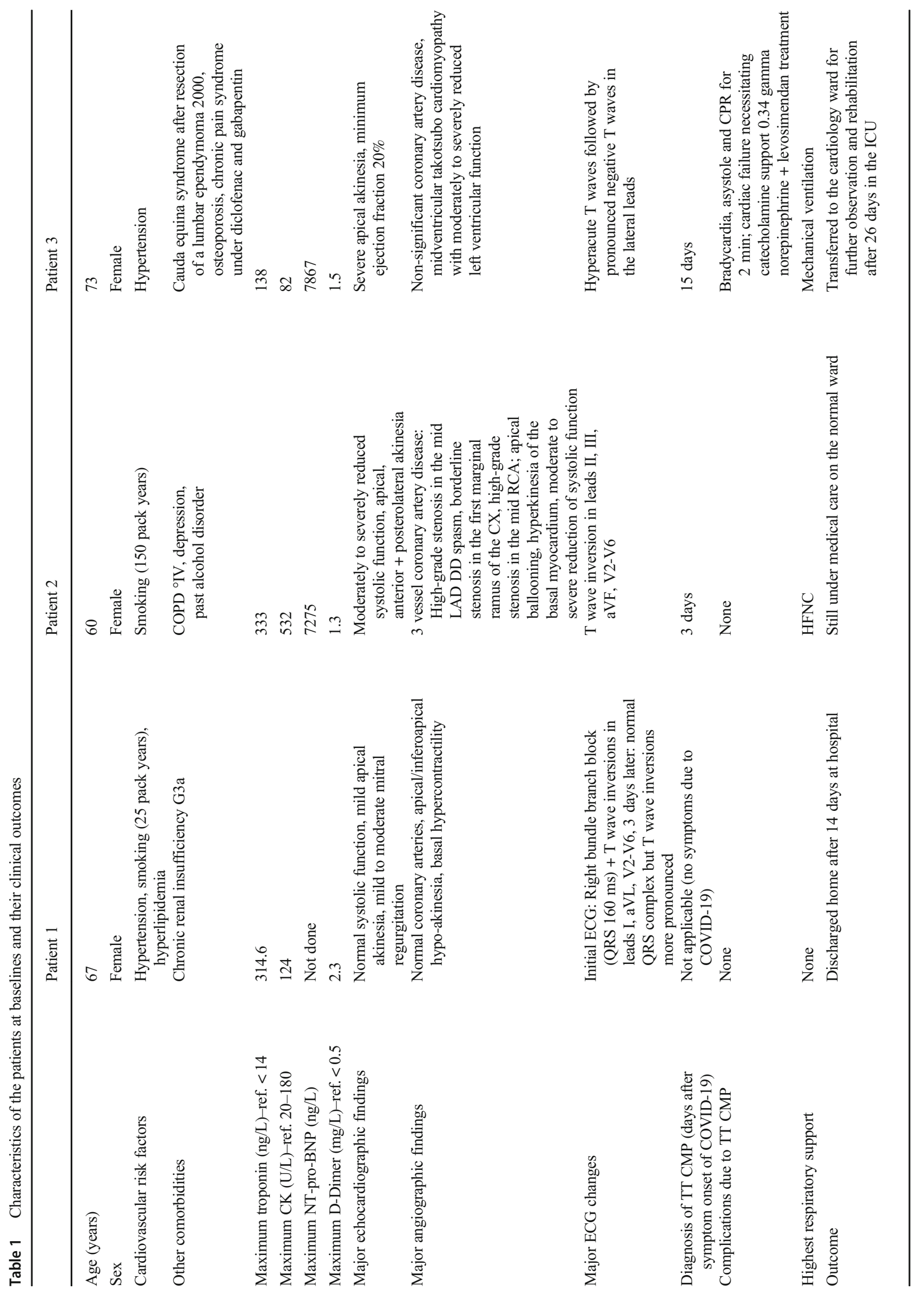


After transfer to the cardiology ward when she was no longer infectious, a follow-up echocardiography proved marked improvement in systolic function (ejection fraction $68 \%)$.

Henceforth, a second CAG was performed 2 weeks after the first one and drug-eluting stents were successfully implanted into the left anterior descending and the circumflex arteries; in a third intervention 5 days later, the right coronary artery was successfully stented.

The diagnosis of TT cardiomyopathy in patient 3 was made while she was already on mechanical ventilation in the intensive care unit because of COVID-19 pneumonia.

As antiviral therapy, she had received remdesivir [12, 13], and dexamethasone for immunomodulation.

Eight days after intubation, the patient suddenly developed bradycardia and asystole and was successfully resuscitated mechanically and chemically (return of spontaneous circulation after $2 \mathrm{~min}$ ).

The ECG after resuscitation was noteworthy for hyperacute $T$ waves, which were later replaced by deep $T$ wave inversions in V3 to V6.

Consecutively, troponin levels rose to a maximum of $130 \mathrm{ng} / \mathrm{L}$, and NT-pro-BNP to $7867 \mathrm{ng} / \mathrm{L}$.

TTE in patient 3 detected severe apical akinesia with hyperkinesia of the basal segments and a minimum ejection fraction (EF) of $20 \%$ after it had been only moderately reduced 2 days earlier.

The patient temporarily required hemodynamic support with up to 0.34 gamma norepinephrine. She received a course of intravenous levosimendan, after which left ventricular function continually improved.

Six days after cardiopulmonary resuscitation, CAG was performed and showed only bystander coronary artery disease

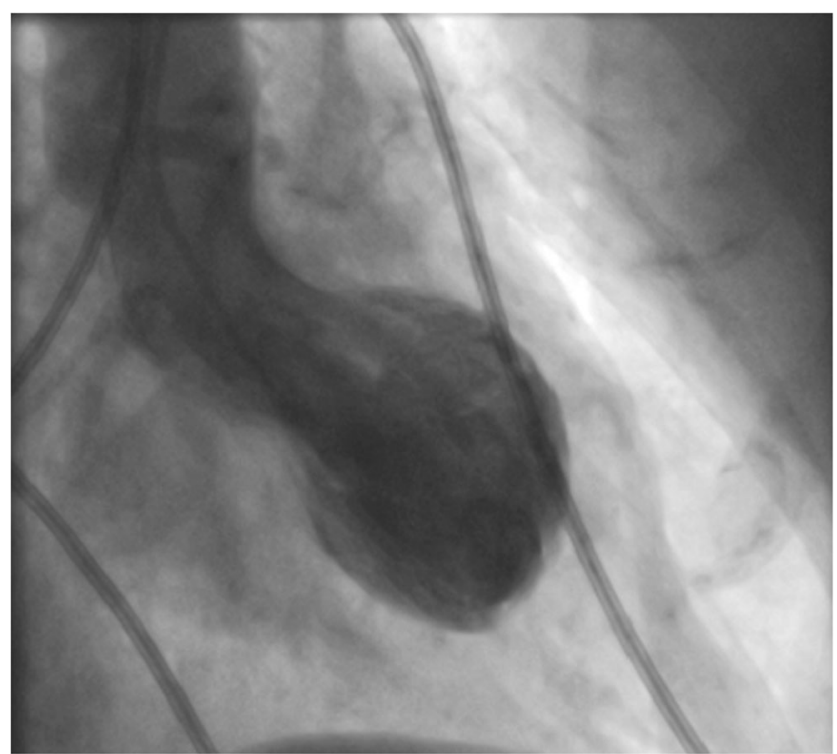

Fig. 1 Classical takotsubo with apical ballooning in patient 2. Left ventriculography in the right anterior oblique protection during systole (non-significant wall irregularities in the mid-left anterior descending and in the right coronary artery). Therefore, the patient was put on aspirin monotherapy.

Furthermore, as far as her respiratory situation was concerned, after a tracheostomy, she could be successfully weaned from the ventilator. Following two negative SARS$\mathrm{CoV}-2$ PCR results, she was no longer considered infectious and could be transferred to a non-COVID ward on $2 \mathrm{~L}$ per minute of oxygen insufflation via a nasal cannula.

A follow-up TTE about 2 weeks after the episode of asystole showed a normal global systolic left ventricular function.

Due to limited resources and in order to minimize infection risk for staff, magnetic resonance imaging to confirm the diagnosis of TT cardiomyopathy and to rule out concomitant myocarditis was not performed in any of the three patients described.

\section{Conclusions}

Contrary to a case series from the USA [14], and in accordance with the predominance of the female sex in takotsubo cardiomyopathy, all three patients in our case series were women in the typical age for manifestation (mean, 67 years). This stands in stark contrast to the fact that severe COVID-19 occurs more frequently in men than in women [15].

However, as has been pointed out in an article by Bentivegna et al., patients may of course recover from COVID-19 despite an extremely unfavorable prognosis [16].

A variety of potential triggers for stress cardiomyopathy have been identified, among which emotional stress plays

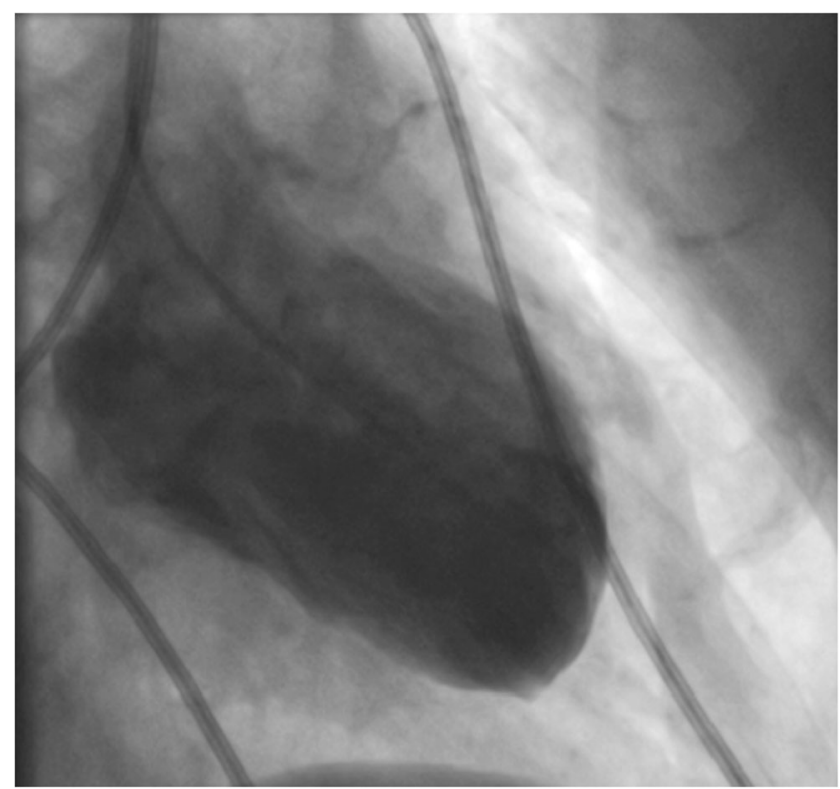

Fig. 2 Classical takotsubo with apical ballooning in patient 2. Left ventriculography in the right anterior oblique protection during diastole 
the most prominent role [10], explaining the synonym of "broken heart syndrome."

The anxieties, worries, and tensions due to the pandemic itself and the associated economic and personal consequences seem to produce a rising incidence in TT cardiomyopathy. This was demonstrated in a retrospective analysis of acute coronary syndromes in five different groups based on the date of their clinical presentation at two Cleveland hospitals with primary cardiac catheterization and intervention capability [17].

The exact mechanisms causing stress cardiomyopathy remain elusive. A number of theories have been suggested, such as a hyperadrenergic state causing a form of myocardial stunning, which is most prominent in the apical sections of the heart due to higher beta-adrenoreceptor density [18].

Alternatively, at least in some patients, a sort of serotonin syndrome causing general stress in patients may be partly responsible for takotsubo cardiomyopathy $[19,20]$.

In our patients, no serotonin syndrome was diagnosed and other triggers for stress cardiomyopathy were more plausible: in patient 1 , it may have been the anxieties associated with the COVID-19 pandemic itself or a tooth extraction 5 days earlier; patient 2 suffered from severe shortness of breath because of high-grade chronic obstructive lung disease exacerbated by COVID-19; and patient 3 was mechanically ventilated in the intensive care unit due to extensive COVID-19 pneumonia.

While takotsubo cardiomyopathy seems to be associated with a lower cardiovascular risk profile than in CAD, which is consistent with our case series, its prognosis is almost as serious as CAD [21].

Indeed, in a large multicenter study, TT cardiomyopathy led to cardiac failure in a substantially higher proportion of patients than an acute coronary syndrome [22].

Contrary to this fact, the prognosis in our small case series was favorable, with the 28-day mortality at $0 \%$.

The major limitation of this case series is, obviously, the small sample size.

Moreover, whether the patients described here developed TT cardiomyopathy as a direct complication of COVID-19 or due to the stress of the pandemic or of their hospitalization is a matter of speculation at this point.

Strengths of this study include the extensive clinical and laboratory follow-up. In contrast to other reports, all three patients underwent coronary angiography, which is the gold standard to rule out an acute coronary syndrome as the major differential diagnosis for takotsubo cardiomyopathy.

Coronary angiography should not be withheld for COVID19 patients if clinically indicated.

In conclusion, this report is intended to raise awareness in both infectious diseases and cardiology specialists for stress cardiomyopathy as a complication in COVID-19. The importance of treating patients infected with SARS-CoV-2 with the same clinical standard as non-infectious patients should be emphasized.
Authors' Contributions All authors collected, analyzed, and interpreted the data.

The first draft of the manuscript was written by Wolfgang Hoepler, and all authors revised the article critically for important intellectual content and gave final approval of the current version to be submitted.

Data Availability All data and material are stored electronically and can be made available upon request.

\section{Compliance with Ethical Standards}

Conflict of Interest The authors declare that they have no competing interests.

Ethics Approval Not applicable, as this is a retrospective analysis with anonymous data.

Consent for Publication As the data presented here do not allow identification of an individual patient, a written consent for publication was not deemed necessary.

Code Availability Not applicable.

\section{References}

1. Spuntarelli V, Luciani M, Bentivegna E, Marini V, Falangone F, Conforti G, et al. COVID-19: Is it just a lung disease? A case-based review. SN Compr Clin Med. 2020:1-6. https://doi.org/10.1007/ s42399-020-00418-6.

2. Shi S, Qin M, Shen B, Cai Y, Liu T, Yang F, et al. Association of cardiac injury with mortality in hospitalized patients with COVID19 in Wuhan, China. JAMA Cardiol. 2020;5(7):802-10. https://doi. org/10.1001/jamacardio.2020.0950.

3. Puntmann VO, Carerj ML, Wieters I, Fahim M, Arendt C, Hoffmann J, et al. Outcomes of cardiovascular magnetic resonance imaging in patients recently recovered from coronavirus disease 2019 (COVID-19). JAMA Cardiol. 2020:1265-73. https://doi. org/10.1001/jamacardio.2020.3557.

4. Rajpal S, Tong MS, Borchers J, Zareba KM, Obarski TP, Simonetti $\mathrm{OP}$, et al. Cardiovascular magnetic resonance findings in competitive athletes recovering from COVID-19 infection. JAMA Cardiol. 2020. https://doi.org/10.1001/jamacardio.2020.4916.

5. Arcari L, Luciani M, Cacciotti L, Musumeci MB, Spuntarelli V, Pistella E, et al. Incidence and determinants of high-sensitivity troponin and natriuretic peptides elevation at admission in hospitalized COVID-19 pneumonia patients. Intern Emerg Med. 2020;15(8): 1467-76. https://doi.org/10.1007/s11739-020-02498-7.

6. Babapoor-Farrokhran S, Gill D, Walker J, Rasekhi RT, Bozorgnia B, Amanullah A. Myocardial injury and COVID-19: Possible mechanisms. Life Sci. 2020;253:117723. https://doi.org/10.1016/ j.lfs.2020.117723.

7. Zheng Y-Y, Ma Y-T, Zhang J-Y, Xie X. COVID-19 and the cardiovascular system. Nat Rev Cardiol. 2020;1-2. https://doi.org/10. 1038/s41569-020-0360-5.

8. Varga Z, Flammer AJ, Steiger P, et al. Endothelial cell infection and endotheliitis in COVID-19. Lancet. 2020;395(10234):1417-8. https://doi.org/10.1016/S0140-6736(20)30937-5.

9. Chen L, Li X, Chen M, Feng Y, Xiong C. The ACE2 expression in human heart indicates new potential mechanism of heart injury among patients infected with SARS-CoV-2. Cardiovasc Res. 2020;116(6):1097-100. https://doi.org/10.1093/cvr/cvaa078. 
10. De Chazal HM, Del Buono MG, Keyser-Marcus L, et al. Stress cardiomyopathy diagnosis and treatment. J Am Coll Cardiol. 2018;72(16):1955-71. https://doi.org/10.1016/j.jacc.2018.07.072.

11. Seitz T, Hoepler W, Weseslindtner L, Aberle JH, Aberle SW, Puchhammer-Stoeckl E, et al. Successful management of the first reported case in Austria of COVID-19 with ARDS. Infection. 2020;48(4):647-51. https://doi.org/10.1007/s15010-020-01458-9.

12. Pennica A, Conforti G, Falangone F, et al. Clinical management of adult coronavirus infection disease 2019 (COVID-19) Positive in the setting of low and medium intensity of care: a short practical review. SN Compr Clin Med. 2020:1-6. https://doi.org/10.1007/ s42399-020-00333-w.

13. Beigel JH, Tomashek KM, Dodd LE, et al. Remdesivir for the Treatment of Covid-19 - Final Report. N Engl J Med. 2020;383(19):1813-26. https://doi.org/10.1056/NEJMoa2007764.

14. Giustino G, Croft LB, Oates CP, Rahman K, Lerakis S, Reddy VY, et al. Takotsubo cardiomyopathy in COVID-19. J Am Coll Cardiol. 2020;76(5):628-9. https://doi.org/10.1016/j.jacc.2020.05.068.

15. Williamson EJ, Walker AJ, Bhaskaran K, Bacon S, Bates C, Morton CE, et al. Factors associated with COVID-19-related death using OpenSAFELY. Nature. 2020;584(7821):430-6. https://doi. org/10.1038/s41586-020-2521-4.

16. Bentivegna E, Luciani M, Spuntarelli V, Speranza ML, Guerritore L, Sentimentale A, et al. Extremely severe case of COVID-19 pneumonia recovered despite bad prognostic indicators: a didactic report. SN Compr Clin Med. 2020:1-4. https://doi.org/10.1007/ s42399-020-00383-0.

17. Jabri A, Kalra A, Kumar A, Alameh A, Adroja S, Bashir H, Nowacki AS, Shah R, Khubber S, Kanaa'N A, Hedrick DP, Sleik
KM, Mehta N, Chung MK, Khot UN, Kapadia SR, Puri R, Reed GW Incidence of stress cardiomyopathy during the coronavirus disease 2019 Pandemic. JAMA Netw Open 2020;3(7). https://doi. org/10.1001/jamanetworkopen.2020.14780.

18. Lyon AR, Rees PSC, Prasad S, Poole-Wilson PA, Harding SE. Stress (takotsubo) cardiomyopathy-a novel pathophysiological hypothesis to explain catecholamine-induced acute myocardial stunning. Nat Clin Pract Cardiovasc Med. 2008;5(1):22-9. https://doi. org/10.1038/ncpcardio1066.

19. Mehta NK, Aurigemma G, Rafeq Z, Starobin O. Reverse takotsubo cardiomyopathy after an episode of serotonin syndrome. Tex Heart Inst J. 2011;38(5):568-72.

20. Sasaki H, Yumoto K, Nanao T, Nishizawa H, Funada S, Aoki H, et al. Cardiogenic shock due to takotsubo cardiomyopathy associated with serotonin syndrome. J Cardiol Cases. 2012;7(1):e1-3. https:// doi.org/10.1016/j.jccase.2012.07.011.

21. Tornvall P, Collste O, Ehrenborg E, Järnbert-Petterson H. A casecontrol study of risk markers and mortality in takotsubo stress cardiomyopathy. J Am Coll Cardiol. 2016;67(16):1931-6. https://doi. org/10.1016/j.jacc.2016.02.029.

22. Templin C, Ghadri JR, Diekmann J, Napp LC, Bataiosu DR, Jaguszewski M, et al. Clinical features and outcomes of takotsubo (stress) cardiomyopathy. N Engl J Med. 2015;373(10):929-38. https://doi.org/10.1056/NEJMoa1406761.

Publisher's Note Springer Nature remains neutral with regard to jurisdictional claims in published maps and institutional affiliations. 